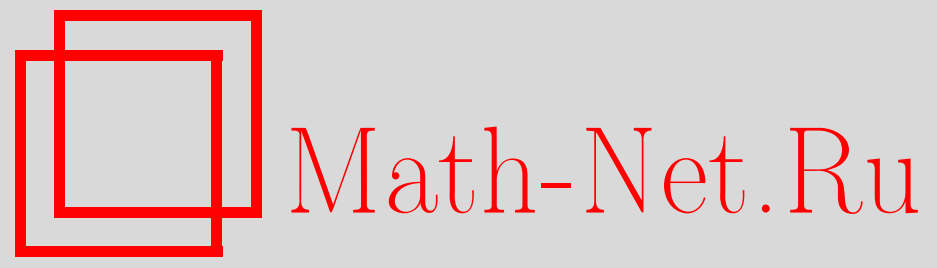

М. А. Иорданский, Структура и способы порождения замкнутых классов графов, Дискрет. матем., 2003, том 15, выпуск 3, 105-116

DOI: https://doi.org/10.4213/dm209

Использование Общероссийского математического портала Math-Net.Ru подразумевает, что вы прочитали и согласны с пользовательским соглашением http://www . mathnet.ru/rus/agreement

Параметры загрузки:

IP : 54.80 .73 .141

26 апреля 2023 г., 14:43:21 


\title{
Структура и способы порождения замкнутых классов графов
}

\author{
() 2003 г. М. А. Иорданский
}

\begin{abstract}
Рассматривается функциональный подход к системе графов с операциями: изучается структура классов графов, замкнутых относительно различных операций, общее число замкнутых классов, число предполных классов, способы порождения классов.

Выделены системы операций, при использовании которых мощность множества замкнутых классов графов соответственно континуальна, счетна или конечна. Показано, что при использовании различных систем операций реализуются все логические возможности для порождения замкнутых классов графов: существуют классы с конечными и счетными базисами, а также классы, не имеющие базиса.

Работа выполнена при подлержке Российского фонда фундаментальных исследований, проект 01-01-00464.
\end{abstract}

\section{1. Введение}

Рассматривается множество $\mathscr{G}$ всех конечных неориентированных графов, допускающих петли и кратные ребра. Для них в статье используется термин граф. Приняты следующие обозначения: $V(G)$ - множество вершин графа $G, K_{n}$ - полный $n$-вершинный обыкновенный граф, $K_{0}-$ нуль-граф, не содержащий вершин; $\bar{G}-$ дополнение обыкновенного графа $G$ до полного; $\bar{K}_{n}-$ пустой $n$-вершинный граф; $K_{\circ}-$ изолированная вершина; $C_{n}$ - простой цикл, содержащий $n \geqslant 1$ вершин, $C_{1}$ - петля; $L_{n}$ - простая цепь, содержащая $n \geqslant 3$ вершин; $Z\left(G_{1}, G_{2}, \ldots, G_{L}\right)$ - класс графов, не содержащих подграфы $G_{1}, \ldots, G_{L} ; S\left(G_{1}, G_{2}, \ldots, G_{L}\right)$ - класс графов, содержащих хотя бы один из подграфов $G_{1}, G_{2}, \ldots, G_{L}$.

К графам применяются операции для построения одних графов из других. Описание порождаемых при этом классов графов производится на основе понятий замыкания и замкнутого класса. Пусть $P$ - некоторое (не обязательно конечное) множество графов. Множество $[P]$ всех графов, получаемых из $P$ с помощью определенной системы операций, образует замыкание $P$. Если $[P]=P$, то $P$ - замкнутый класс графов. При описании способов порождения замкнутых классов используется понятие базиса - минимального по включению множества графов, замыкание которого дает весь класс. Замкнутый класс $R_{1} \subset R_{2}$ называется предполным [1] в замкнутом классе $R_{2}$, если его замыкание $\left[R_{1}\right] \neq R_{2}$, но при добавлении к $R_{1}$ любого элемента $r \in R_{2} \backslash R_{1}$ замыкание $\left[R_{1} \cup r\right]=R_{2}$. Предполный класс $R_{1}$ называется тривиальным, если множество $R_{2} \backslash R_{1}$ содержит ровно один элемент. 
Операция сохраняет некоторое свойство исходного графа (графов), если им обладает и результирующий граф. Система операций сохраняет некоторое свойство графов, если его сохраняют все операции системы. Число различных сохраняемых свойств, характеризует силу системы операций: чем меньше сохраняемых свойств, тем сильнее система операций и наоборот. Поскольку каждому сохраняемому свойству соответствует свой замкнутый класс графов, то, иначе говоря, система операций тем сильнее, чем меньше замкнутых классов графов она порождает.

Примером самой сильной системы являются операции добавления (удаления) ребра, петли или изолированной вершины. Они позволяют получать каждый граф из каждого, сохраняя лишь свойство быть графом. При этом имеегся единственный замкнутый класс, совпадающий с множеством всех графов, и каждый граф образует его базис.

Все операции над графами можно разбить на три группы [2]:

(1) Операции композиции (сборки), при выполнении которых из нескольких графов образуется новый граф, в каком-то смысле более сложный, чем каждый из исходных. К таким операциям относятся, например, операции объединения, соединения и произведения графов.

(2) Операции разборки, когда данный граф превращается в несколько графов, каждый из которых в некотором смысле проще исходного. В качестве примера здесь можно привести операции удаления ребра или вершины вместе с инцидентными ребрами (петлями). Эти операции являются обратными по отношению к операциям сборки.

(3) Операции преобразования, не усложняющие и не упрощающие граф. К таким операциям относятся, например, операции стягивания ребра или расщепления вершины.

В работе рассматривается система операций, включающая в себя представителей из всех трех указанных групп, позволяющая получать произвольный граф из каждого непустого графа (теорема 1) или, иначе говоря, реализовывать любую операцию над непустыми графами. При сравнении графов по сложности более простым считается граф, изоморфный собственному подграфу другого графа.

В качестве операций сборки используются операции склейки [3]. Пусть $G_{1}$ и $G_{2}$ - непересекающиеся графы, то есть $V\left(G_{1}\right) \cap V\left(G_{2}\right)=\varnothing$, содержащие изоморфные подграфы $G_{1}^{\prime} \subseteq G_{1}$ и $G_{2}^{\prime} \subseteq G_{2}$. Объединение графов $G_{1}$ и $G_{2}$ путем отождествления их изоморфных подграфов $G_{1}^{\prime} \subseteq G_{1}$ и $G_{2}^{\prime} \subseteq G_{2}$ реализует операцию бинарной склейки графов $G_{1}$ и $G_{2}$. Операция бинарной склейки называется тривиальной, если $G_{1}^{\prime}=G_{1}$ или $G_{2}^{\prime}=G_{2}$ и результирующий граф изоморфен хотя бы одному из графов-операндов.

В качестве операций разборки используются операции бинарной расклейки - обратные к операциям бинарной склейки.

К операциям преобразования относятся операции унарной склейки, при выполнении которых отождествляются изоморфные подграфы $G^{\prime}$ и $G^{\prime \prime}$ одного и того же графа $G$. Отождествляемые подграфы могут пересекаться при условии, что найдется автоморфизм их подграфа пересечения, который можно продолжить до изоморфизма подграфов $G^{\prime}$ и $G^{\prime \prime}$. При выполнении тривиальной операции унарной склейки исходный и результирующий графы изоморфны между собой. К операциям преобразования также относятся операции унарной расклейки, обратные к операциям унарной склейки.

Тривиальные операции склейки являются примером самой слабой системы операций. Они сохраняют все свойства графов. При этом каждое подмножество графов образует замкнутый класс. Всего имеется континуум замкнутых классов, счетные подмножества которых являются тривиальными предполными классами. 
Рассматривается семейство всевозможных операций бинарной склейки над элементами множества $\mathscr{G} \times \mathscr{G}$ и операций унарной склейки над $\mathscr{G}$. Пусть отождествляемые подграфы операции склейки изоморфны графу $\tilde{G}$. Для результирующего графа операции склейки графов $G_{1}$ и $G_{2}$ по $\tilde{G}$ используется запись $\left(G_{1} \circ G_{2}\right) \tilde{G}$; для результирующего графа операции унарной склейки используется обозначение $(G) \tilde{G}$.

При фиксированных графах-операндах результат операции склейки в общем случае зависит от вида отождествляемых подграфов, их выбора и способа отождествления [3]. При наличии определенных симметрий в графах-операндах и в отождествляемых подграфах формулы $\left(G_{1} \circ G_{2}\right) \tilde{G}$ и $(G) \tilde{G}$, а также их суперпозиции задают графы с точностью до изоморфизма и используются в качестве языка задания графов.

Для различных подмножеств операций склейки и расклейки устанавливается общее число замкнутых классов и число предполных классов. Рассматриваются способы порождения классов, наличие или отсутствие базисов, их количество и мощность.

Замечание 1. Нуль-граф $K_{0}$ образует тривиальный замкнутый класс относительно любых операций. Этот класс не будет учитываться в дальнейшем.

\section{2. Операции склейки и расклейки}

Теорема 1. Относительно операчий склейки и расклейки замкнуто лишь два класса гра-

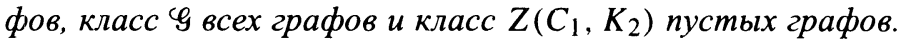

Доказательство. Класс $Z\left(C_{1}, K_{2}\right)$ пустых графов замкнут относительно рассматриваемых операций, поскольку они сохраняют, очевидно, отсутствие в графах ребер и петель.

Покажем, что класс $Z\left(C_{1}, K_{2}\right)$ является единственным предполным в классе $\mathscr{G}$ всех графов. Для этого достаточно установить, что из произвольного графа, не являющегося пустым, можно построить любой граф $G$. С помощью операций бинарной и унарной расклейки и склейки нетрудно получить графы $K_{1}, C_{1}$ и $K_{2}$. Из этих графов с помощью операций бинарной склейки можно получить граф $G$, например, так:

(1) построить пустой $|V(G)|$-вершинный граф с помощью $|V(G)|-1$ операций склейки по $K_{0}$, реализующих графы вида $\left(g \circ K_{1}\right) K_{0}$, где $g$ результирующий граф предыдущей операции склейки ( $g=K_{1}$ при выполнении первой операции);

(2) дополнить пустой граф ребрами (петлями) до графа $G$, использовав $|E(G)|$ операций склейки, реализующих графы вида $\left(g \circ K_{2}\right) \bar{K}_{2}\left(\left(g \circ C_{1}\right) K_{1}\right)$.

Поскольку из каждого пустого графа с помощью операций унарной склейки или расклейки по $K_{1}$ можно построить любой пустой граф, то в классе $Z\left(C_{1}, K_{1}\right)$ нет собственных замкнутых подклассов.

Теорема 1 доказана.

Следствие 1. При использовании операций склейки и расклейки классы $\mathscr{G}$ и $Z\left(C_{1}, K_{2}\right)$ имеют счетное число одноэлементных базисов. 


\section{3. Операции склейки и удаления петли}

Ограничение операций расклейки операцией удаления петли не влияет существенно на структуру замкнутых классов.

Теорема 2. Относительно операчий склейки и удаления петли замкнуто лишь три класса графов, класс $\mathscr{G}$ всех графов, класс $Z\left(K_{2}\right)$ графов, не содержаших ребер, и класс $Z\left(C_{1}, K_{2}\right)$ nустых графов.

Доказательство. Система операций склейки и удаления петли сохраняет такие свойства графов, как отсутствие ребер (класс $\left.Z\left(K_{2}\right)\right)$ и отсутствие петель и ребер (класс $Z\left(C_{1}, K_{2}\right)$ ). Последний класс не содержит собственных замкнутых подклассов, поскольку все пустые графы могут быть получены друг из друга с помощью операций унарной и бинарной склейки по $K_{1}$. Класс $Z\left(C_{1}, K_{2}\right)$ является единственным предполным классом в $Z\left(K_{2}\right)$, так как из любого графа $G \in Z\left(K_{2}\right) \backslash Z\left(C_{1}, K_{2}\right)$ можно построить произвольный граф, не содержащий ребер, используя операции склейки и удаления петли. В свою очередь класс $Z\left(K_{2}\right)$ является единственным предполным классом в $\mathscr{G}$, поскольку из любого графа $G \in \mathscr{G} \backslash Z\left(K_{2}\right)$ с помощью операций унарной склейки и удаления петли можно получить графы $K_{2}, C_{1}$ и $K_{1}$. Из этих графов можно построить любой граф с помощью операций бинарной склейки, например, так, как это сделано в доказательстве теоремы 1. Пооккольку класс $\mathscr{G}$ замкнут относительно любых операций над графами, в целом получаем три замкнутых класса графов.

Теорема 2 доказана.

Следствие 2. При использовании операчий склейки и удаления петли классы $\mathscr{G}, Z\left(K_{2}\right)$ и $Z\left(C_{1}, K_{2}\right)$ имеют счетное число одноэлементных базисов.

\section{4. Операции бинарной склейки и удаления ребра или петли}

Замена операций унарной склейки на операцию удаления ребра приводит к качественному изменению структуры замкнутых классов - переходу от конечного к счетному числу замкнутых классов. Приведем два вспомогательных утверждения.

Лемма 1. Из любого п-вершинного графа, содержсащего ребро и петлю, можно построить произвольный граф, содержащий не менее $n$ вершин, используя операчии бинарной склейки и удаления ребра или петли.

Доказательство. С помощью операций удаления ребра или петли можно построить пустой граф, а также два графа, один из которых содержит единственное ребро, а другой единственную петлю, сохранив число вершин, равным $n$. Используя операции бинарной склейки по $\bar{K}_{n}$, из двух последних графов строится любой непустой $n$-вершинный граф. Для построения из трех указанных $n$-вершинных графов произвольного графа с числом вершин, большим $n$, достаточно использовать операции бинарной склейки по $\bar{K}_{r}, r<n$.

Лемма 1 доказана. 
Следствие 3. Из любого непустого п-вершинного графа, не содержашего петель, можно построчть произвольный граф без петель с числом вершин, не меньшим $n$, используя операчии бинарной склейки и удаления ребра.

Следствие 4. Из любого непустого п-вершинного графа, не содержащего ребер, можно построчть произвольный граф без ребер с числом вершин, не меньшим $n$, используя операчии бинарной склейки и удаления петли.

Лемма 2. Каждый класс графов, замкнутый относительно операчий бинарной склейки и удаления ребра или петли, имеет базис, содержащий не более трех элементов.

Доказательство. Если среди графов с минимальным числом вершин содержится граф с ребром и петлей, то в силу леммы 1 он один образует базис. Если граф с минимальным числом вершин является пустым, то возможны следуюшие ситуации:

- если класс содержит только пустые графы, то его базис состоит из этого графа;

- если среди непустых графов с минимальным числом вершин найдется граф, содержащий ребро и петлю, то базис содержит этот граф и исходный пустой;

- если все непустые графы с минимальным числом вершин содержат только ребра (петли), то базис состоит не более чем из трех графов, пустого графа, графа, содержашего только ребра (петли), а также, быть может, графа с петлей (ребром);

- если непустые графы с минимальным числом вершин содержат только ребра или только петли, то базис состоит из трех графов, пустого графа, графа без петель и графа без ребер.

Все графы, включенные в базис, имеют минимальное число вершин среди графов соответствующего вида.

Лемма 2 доказана.

Следствие 5. Класс $Z\left(C_{1}, K_{2}\right)$ пустых графов, замкнутый относительно операчий бинарной склейки и удаления ребра или петли, имеет единственный базис $B_{1}=\left\{K_{1}\right\}$. Все другие классы, замкнутые относительно указанных операчий, имеют счетное число базисов.

Графы, принадлежащие разным базисам, различаются числом кратных ребер или петель.

Теорема 3. Мощность множества всех классов графов, замкнутых относительно операчий бинарной склейки и удаления ребра или петли, счетна.

Доказательство. Найдем оценку снизу. Все используемые операции не сокращают число вершин в графах. Таким образом, имеем счетное число замкнутых классов графов, каждый из которых содержит графы с числом вершин, не меньшим заданного.

Получим оценку сверху. В силу леммы 2 число рассматриваемых замкнутых классов не превосходит числа всех трехэлементных подмножеств счетного множества графов и, следовательно, имеет счетную мощность.

Теорема 3 доказана. 


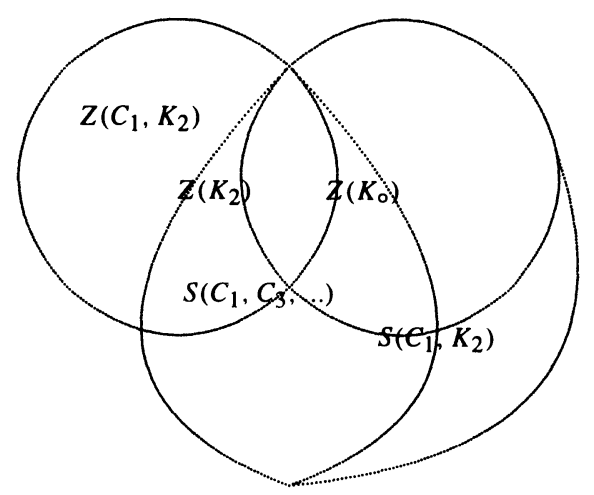

Рис. 1.

Обозначим через $\mathscr{G}_{C_{1}}^{1}$ множество всех одновершинных графов, содержащих петлю, и через $\mathscr{G}_{K_{2}}^{2}$ множество всех двухвершинных графов, содержащих ребро. Нетрудно видеть, что классы графов $\mathscr{G} \backslash \mathscr{G}_{C_{1}}^{1}$ и $\mathscr{G} \backslash \mathscr{G}_{K_{2}}^{2}$ замкнуты относительно операций бинарной склейки и удаления ребра или петли.

Теорема 4. При использовании операчий бинарной склейки и удаления ребра или петли в множестве $\mathscr{G}$ всех графов имеется лишь два предполных класса $\mathscr{G} \backslash \mathscr{G}_{C_{1}}^{1} u \mathscr{G} \backslash \mathscr{G}_{K_{2}}^{2}$.

Доказательство. Предполнота класса $\mathscr{G} \backslash \mathscr{G}_{C_{1}}^{1}$ следует из того, что в множестве $\mathscr{G}_{C_{1}}^{1}$ каждый граф может быть получен из каждого с помощью рассматриваемых операций. Предполнота класса $\mathscr{G} \backslash \mathscr{G}_{K_{2}}^{2}$ обусловлена тем, что из любого графа, принадлежащего $\mathscr{G}_{K_{2}}^{2}$, быть может, графа $C_{1} \subset \mathscr{G} \backslash \mathscr{G}_{K_{2}}^{2}$ можно построить произвольный граф из $\mathscr{G}_{K_{2}}^{2}$ с помощью операций бинарной склейки и удаления ребра или петли.

Любой другой предполный класс графов должен содержать хотя бы по одному представителю из множеств $\mathscr{G}_{C_{1}}^{1}$ и $\mathscr{G}_{K_{2}}^{2}$. Используя операции бинарной склейки и удаления ребра или петли, из произвольного графа множества $\mathscr{G}_{C_{1}}^{1}$ можно построить графы $C_{1}$ и $K_{1}$, а из любого графа множества $\mathscr{G}_{K_{2}}^{2}-$ граф $K_{2}$. Так как с помощью операций бинарной склейки из системы $\left\{K_{1}, C_{1}, K_{2}\right\}$ можно построить все графы (в силу теоремы 1 ), других предполных классов в $\mathscr{G}$ нет.

Теорема 4 доказана.

\section{5. Операции склейки}

Из определения операций склейки следует, что они сохраняют отсутствие изолированных вершин (класс $\left.Z\left(K_{\circ}\right)\right)$ и отсутствие ребер (класс $Z\left(K_{2}\right)$ ). Нетрудно видеть, что операции склейки сохраняют также одновременное отсутствие петель и ребер (класс $Z\left(C_{1}, K_{2}\right)$ пустых графов) или наличие петель или ребер (класс $S\left(C_{1}, K_{2}\right)$ непустых графов). Далее нам понадобится также следующее утверждение. 
Лемма 3. Операчии склейки сохраняют наличие в графах чикла нечетной длины.

Доказательство. Операции бинарной склейки сохраняют все циклы графов-операндов. Любая операция унарной склейки может быть реализована последовательностью операций, в каждой из которых отождествляется пара вершин или пара кратных ребер (петель) текущего графа. Очевидно, что склейки кратных ребер (петель) сохраняют в графе цикл нечетной длины.

Рассмотрим отождествление пар вершин. Если нечетному циклу принадлежит лишь одна вершина из отождествляемой пары, то длина цикла не изменится. При отождествлении двух вершин нечетного цикла образуется два пореберно непересекающихся цикла, сумма длин которых равна длине исходного цикла, то есть нечетна. Следовательно, один из новых циклов имеет нечетную длину.

Лемма 3 доказана.

Следствие 6. Класс $S\left(C_{1}, C_{3}, \ldots\right)$ графов, содержащих цикл нечетной длины, замкнут относительно операчий склейки.

Взаимное расположение всех вышеуказанных замкнутых классов приведено на рис. 1.

Лемма 4. Класс $S\left(C_{1}, K_{2}\right)$ непустых графов, замкнутый относительно операчий склей-

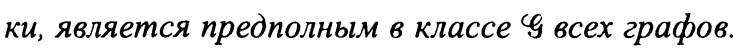

Доказательство. В $Z\left(C_{1}, K_{2}\right)=\varphi \backslash S\left(C_{1}, K_{2}\right)$ каждый граф может быть получен из каждого с помощью операций унарной и бинарной склейки, то есть любой граф $G \in$ $Z\left(C_{1}, K_{2}\right)$ образует базис класса $Z\left(C_{1}, K_{2}\right)$. Следовательно, $\left[S\left(C_{1}, K_{2}\right) \cup G\right]=\varphi$.

Лемма 4 доказана.

Лемма 5. Из любого непустого графа $G$, не содержащего чикл нечетной длины, можно построить граф $K_{2}$, используя операчии унарной склейки.

Доказательство. Доказательство проведем индукцией по числу ребер графа. Если граф $G$ содержит одно ребро, но не совпадает с $K_{2}$, то граф $K_{2}$ строится с помощью операций, реализующих графы вида $(g) K_{1}$, где $g$ - текущий граф (вначале $g=G$ ) и хотя бы одна из отождествляемых вершин является изолированной.

Пусть из каждого непустого графа, не имеющего цикла нечетной длины и содержащего не более $m$ ребер, можно построить граф $K_{2}$. Рассмотрим произвольный граф $G$ с $m+1$ ребром, не содержащий цикл нечетной длины. Если в $G$ имеются изолированные или кратные ребра, то среди графов $(G) K_{2}$, очевидно, найдется граф с $m$ ребрами, не содержащий нечетный цикл. В противном случае среди графов $(G) K_{2}$ найдется граф, полученный в результате отождествления пары смежных ребер. Покажем, что при этом не появится цикл нечетной длины. Это очевидно, если хотя бы одно из отождествляемых ребер не входит ни в один цикл. Длина каждого четного цикла, содержащего два смежных отождествляемых ребра, сократится на два, то есть останется четной. Длина каждого четного цикла, содержащего лишь одно ребро из отождествляемой пары, не изменится, а все новые циклы будут иметь четную длину, равную сумме двух нечетных чисел. Таким образом, получаем граф с $m$ ребрами, не содержащий цикл нечетной длины, из которого по предположению индукции можно построить граф $K_{2}$.

Лемма 5 доказана. 
Лемма 6. Класс $Z\left(C_{1}, K_{2}\right) \cup S\left(C_{1}, C_{3}, \ldots\right)$ замкнут относительно операчий склейки и

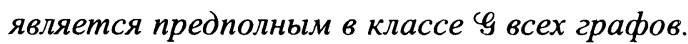

Доказательство. Класс $Z\left(C_{1}, K_{2}\right) \cup S\left(C_{1}, C_{3}, \ldots\right)=Z\left(K_{2}\right) \cup S\left(C_{1}, C_{3}, \ldots\right)$ замкнут относительно операций склейки, поскольку в $S\left(C_{1}, C_{3}, \ldots\right)$ содержатся графы с изолированными вершинами. Его дополнение до класса $\mathscr{G}$ всех графов состоит из всех непустых графов, не содержащих цикл нечетной длины. По лемме 5 из каждого такого графа $G$ можно построить граф $K_{2}$. Класс $Z\left(C_{1}, K_{2}\right) \cup S\left(C_{1}, C_{3}, \ldots\right)$ содержит графы $C_{1}$ и $K_{1}$. Таким образом,

$$
\left[\left(Z\left(C_{1}, K_{2}\right) \cup S\left(C_{1}, C_{3}, \ldots\right)\right) \cup G\right]=\mathscr{G}
$$

поскольку, как уже ранее отмечалось, из графов $C_{1}, K_{1}$ и $K_{2}$ можно построить любой граф.

Лемма 6 доказана.

Лемма 7. Класс графов $Z\left(K_{\circ}\right) \cup S\left(C_{1}, C_{3}, \ldots\right)$ замкнут относительно операчий склейки и является единственным предполным в классе $S\left(C_{1}, K_{2}\right)$ непустых графов.

Доказательство. Склейки графов из $Z\left(K_{\circ}\right) \backslash S\left(C_{1}, C_{3}, \ldots\right)$ с графами из $S\left(C_{1}, C_{3}, \ldots\right) \backslash Z\left(K_{\circ}\right)$ порождают лишь графы, содержащие цикл нечетной длины. Все такие графы уже содержатся в классе $S\left(C_{1}, C_{3}, \ldots\right)$. Таким образом, класс $Z\left(K_{\circ}\right) \cup S\left(C_{1}, C_{3}, \ldots\right)$ является замкнутым относительно операций склейки.

Для доказательства того, что этот класс является единственным предполным в $S\left(C_{1}, K_{2}\right)$ достаточно показать, что из любого непустого графа $G$, не принадлежащего классу $Z\left(K_{\circ}\right) \cup S\left(C_{1}, C_{3}, \ldots\right)$, можно построить произвольный граф из $S\left(C_{1}, K_{2}\right)$. Учитывая лемму 5 , получаем из $G$ граф $K_{2}$. Далее, с помощью операций, реализующих графы вида $\left(g \circ K_{2}\right) \tilde{G}$, где $g$ - текущий граф (вначале $\left.g=K_{2}\right)$ и $\tilde{G}$ есть $K_{0}$ или $K_{1}$, можно построить граф, содержащий порожденный подграф, изоморфный остовному лесу (дереву) искомого графа, а затем добавить к этому подграфу закорачивающие ребра с помощью операций, реализующих графы вида $\left(g \circ K_{2}\right) \bar{K}_{2}$. Необходимое число изолированных вершин устанавливается с помощью операций, реализующих графы вида $(g) K_{1}$ или $(g \circ g) \tilde{G}$, где $\tilde{G}=g \backslash K_{\text {。 }}$.

Лемма 7 доказана.

Лемма 8. Класс $\mathscr{G}$ всех графов содержит только два предполных класса $S\left(C_{1}, K_{2}\right)$ и $Z\left(C_{1}, K_{2}\right) \cup S\left(C_{1}, C_{3}, \ldots\right)$, замкнутых относительно операчий склейки.

Доказательство. Так как указанные классы графов пересекаются по $S\left(C_{1}, C_{3}, \ldots\right)$, любой другой предполный класс должен был бы содержать графы $G_{1} \in S\left(C_{1}, K_{2}\right) \backslash S\left(C_{1}, C_{3}, \ldots\right), G_{2} \in Z\left(C_{1}, K_{2}\right)$. В силу леммы 5 из графа $G_{1}$ можно построить граф $K_{2}$ с помощью операций унарной склейки, а затем граф $C_{1}=\left(K_{2}\right) K_{1}$. Из произвольного графа $G_{2} \in Z\left(C_{1}, K_{2}\right)$ получаем граф $K_{1}$, используя операции унарной склейки. Так как из графов $K_{1}, C_{1}$ и $K_{2}$ строится любой граф (в силу теоремы 1$)$, класс, содержащий графы $G_{1}$ и $G_{2}$, не может быть предполным в $\mathscr{G}$.

Лемма 8 доказана. 
Теорема 5. Мочность множества всех классов графов, замкнутых относительно операчий склейки, континуальна.

Доказательство. Оценка сверху следует из того, что число замкнутых классов графов не превосходит числа всех подмножеств счетного множества $\mathscr{G}$ всех графов. Для установления нижней оценки достаточно выделить бесконечную последовательность графов, каждый член которой не может быть получен из других членов с помощью операций унарной и бинарной склейки. Примером такой последовательности являются графы $G_{i}=\left(C_{2 i-1} \circ K_{2 i-1}\right) K_{0}, i=2,3, \ldots$ Действительно, каждый граф $G_{i}$ не может быть получен с использованием графов $G_{j}, j>i$, поскольку при этом обязательно потребуется склейка смежных вершин (для сокрашения числа вершин в компоненте связности $K_{2 j-1}$ графа $G_{j}$ ) и в текушем графе появится петля, удалить которую в дальнейшем с помощью операций склейки невозможно. С другой стороны, граф $G_{i}$ не может быть получен из графов $G_{j}, j<i$, так как при выполнении над ними любой операции склейки каждая компонента связности результирующего графа будет содержать цикл $C_{2 j-1}, j<i$, а в графе $G_{i}$ одной из компонент связности является цикл $C_{2 i-1}$. Множество замкнутых классов графов, порожденных всеми подмножествами рассмотренной последовательности графов, имеет континуальную мощность.

Теорема 5 доказана.

Существуют классы графов, замкнутые относительно операций склейки, не имеющие базиса. В качестве примера рассмотрим класс $S\left(C_{1}, C_{3}, \ldots\right)$ графов, содержащих нечетный цикл. В нем можно выделить счетное множество замкнутых подклассов $S\left(C_{1}, C_{3}, \ldots, C_{2 i-1}\right), i=1,2, \ldots$, в каждый из которых включены все графы, содержащие цикл нечетной длины из диапазона от 1 до $2 i-1$. Замкнутость этих классов следует из доказательства леммы 3.

Лемма 9. Граф

$$
g_{i}=\left(\left(K_{1} \circ K_{2}\right) K_{0} \circ C_{2 i-1}\right) K_{0}, \quad i=1,2, \ldots
$$

образует базис класса $S\left(C_{1}, C_{3}, \ldots, C_{2 i-1}\right)$, замкнутого относительно операчий склей$\kappa и$.

Доказательство. Доказательство проведем индукцией по $i$. Пусть $i=1$. Покажем, что из графа

$$
g_{1}=\left(\left(K_{1} \circ K_{2}\right) K_{0} \circ C_{1}\right) K_{0}
$$

можно получить любой граф, содержащий петли.

Вначале построим граф, содержащий $|V(G)|$ изолированных вершин, используя операции, реализующие графы вида $\left(g \circ g_{1}\right) \tilde{G}$, где $g$ - текущий граф (вначале $\left.g=g_{1}\right)$ и $\tilde{G}=\left(C_{1} \circ K_{2}\right) K_{0}$.

Затем соединим изолированные вершины ребрами таким образом, чтобы получить при этом граф, изоморфный подграфу графа $G$, не содержащему петель и изолированных вершин. Это делается с помощью операций, реализующих графы вида $\left(g \circ g_{1}\right) \tilde{G}$, где $g$ - текущий граф, $\tilde{G}=\bar{K}_{2}$ и отождествляемые вершины смежны в $g_{1}$. Образующиеся при 
этом дополнительные петли и изолированные вершины склеиваются затем в одну петлю операциями унарной склейки текущего графа по $C_{1}$ или $K_{1}$ соответственно.

Эта петля может быть перенесена на любую вершину графа, инцидентную ребру, или сохранена в виде изолированной петли (результирующий граф $G$ содержит хотя бы одну петлю). При наличии в графе $G$ более одной петли все они склеиваются с помощью операций, реализующих графы вида $\left(g \circ C_{1}\right) K_{1}$. Граф $C_{1}$ можно построить из $g_{1}$ с помощью операций унарной склейки.

Так как

$$
S\left(C_{1}, C_{3}, \ldots, C_{2 i-1}\right)=S\left(C_{1}, C_{3}, \ldots, C_{2 i-3}\right) \cup S\left(C_{2 i-1}\right)
$$

и граф $g_{i-1} \Leftarrow\left(g_{i}\right) K_{2}$, для доказательства индуктивного перехода достаточно показать, что из графа

$$
g_{i}=\left(\left(K_{1} \circ K_{2}\right) K_{0} \circ C_{2 i-1}\right) K_{0}
$$

можно получить любой граф $G$, принадлежащий $S\left(C_{2 i-1}\right)$.

С использованием операций, реализующих графы вида $\left(g \circ g_{i}\right) \tilde{G}$, где $g$ - текущий граф (вначале $\left.g=g_{i}\right)$ и $\tilde{G}=\left(K_{1} \circ C_{2 i-1}\right) K_{0}\left(K_{1}\right.$ совпадает с изолированной вершиной в $\left.g_{i}\right)$, к графу $g_{i}$ добавляется столько изолированных ребер, сколько нетривиальных компонент связности содержит граф $G$.

Далее с помощью операций бинарной склейки по $\tilde{G}=\left(\bar{K}_{2} \circ C_{2 i-1}\right) K_{0}$, где одна вершина из $\bar{K}_{2}$ совпадает с изолированной вершиной в $g_{i}$, для каждой нетривиальной компоненты связности графа $G$ строится ее остовное дерево.

Затем к остовным деревьям добавляются ребра, закорачивающие все циклы $C_{n}, n \geqslant 2$, графа $G$ с использованием склейки по $\tilde{G}=\left(\bar{K}_{3} \circ C_{2 i-1}\right) K_{0}$.

Полученный граф $g$ содержит порожденный подграф, изоморфный подграфу графа $G$ без изолированных вершин и петель. В графе $g$ содержатся также изолированная вершина и цикл $C_{2 i-1}$ из исходного графа $g_{i}$. Их можно удалить с помощью операции унарной склейки по $\left(K_{1} \circ C_{2 i-1}\right) K_{0}$. Добавить необходимое число изолированных вершин можно, используя операции, реализуюшие графы вида $\left(g \circ g_{i}\right) \tilde{G}$, где $\tilde{G}=\left(K_{2} \circ C_{2 i-1}\right) K_{0}$. Так как из графа $g_{i}$ можно построить петлю $C_{1}$ с помощью операций унарной склейки, все необходимые петли добавляются с помощью операций, реализующих графы вида $\left(g \circ C_{1}\right) K_{1}$, где $g-$ текуший граф.

Лемма 9 доказана.

Теорема 6. Замкнутый относительно операчий склейки класс $S\left(C_{1}, C_{3}, \ldots\right)$ арафов, содержащих иикл нечетной длины, не имеет базиса.

Доказательство. Предположим, что класс $S\left(C_{1}, C_{3}, \ldots\right)$ имеет базис $B$ и граф $G \in B$. Пусть $2 i-1, i=1,2, \ldots$, - наибольшая длина нечетных циклов графа $G$, то есть граф $G \in S\left(C_{1}, C_{3}, \ldots, C_{2 i-1}\right)$. Так как классы $S\left(C_{1}, \ldots, C_{2 i-1}\right), i=1,2, \ldots$, замкнуты, в базисе $B$ должна содержаться также некоторая система графов $\left\{G_{1}, G_{2}, \ldots, G_{t}\right\}, t \geqslant 1$, из которой можно получить все графы из $S\left(C_{1}, C_{3}, \ldots, C_{2 i+1}\right) \backslash S\left(C_{1}, C_{3}, \ldots, C_{2 i-1}\right)$. Это множество содержит граф $g_{i+1}=\left(\left(K_{1} \circ K_{2}\right) K_{0} \circ C_{2 i+1}\right) K_{0}$, из которого можно получить граф $g_{i} \Leftarrow\left(g_{i+1}\right) K_{2}$, образующий по лемме 9 базис класса $S\left(C_{1}, C_{3}, \ldots, C_{2 i-1}\right)$. Так как $G \in S\left(C_{1}, C_{3}, \ldots, C_{2 i-1}\right)$, отсюда следует, что из $\left\{G_{1}, G_{2}, \ldots, G_{t}\right\} \subset B$ можно получить граф $G \in B$, что противоречит определению базиса. 
Теорема 6 доказана.

Лемма 10. Если класс графов, замкнутый относительно операчий склейки, имеет базис, то число базисов счетно.

Доказательство. Пусть $G$ - произвольный граф. Среди всех графов, реализуемых нетривиальными операциями склейки $(G \circ G) \tilde{G}$, содержатся графы, при получении которых использовался выбор и отождествление подграфов, изоморфных $\tilde{G}$, согласующиеся с некоторой тривиальной операцией бинарной склейки графов $G$. Применяя к любому такому графу операцию унарной склейки, соответствующую завершению выполнения тривиальной операции бинарной склейки графов $G$, получаем исходный граф $G$.

Если граф $G$ принадлежит базису некоторого замкнутого класса, то его базисами будут и другие системы графов, в которых граф $G$ заменен на один из графов вида $\left.\left(\ldots(G \circ G) \tilde{G}_{1} \circ G\right) \tilde{G}_{2} \circ \ldots \circ G\right) \tilde{G}_{i} \circ \ldots$, при построении которого использовался выбор и отождествление подграфов, изоморфных $\tilde{G}_{j}, j=1,2, \ldots$, согласующиеся с тривиальными операциями склейки текущего графа с графом $G$.

Лемма 10 доказана.

Таким образом, переход к использованию лишь операций склейки существенно увеличивает как число замкнутых классов, так и разнообразие способов их порождения.

Как указывалось во введении, число классов графов, замкнутых относительно различных систем операций, может служить основанием для сравнения операций по силе. Из теорем 2, 3 и 5 получаем следующее утверждение.

Следствие 7. Операчии унарной склейки сильнее операчий удаления ребра, но слабее операчий удаления ребра или петли.

При использовании лишь операций бинарной склейки все замкнутые классы графов имеют единственный конечный или счетный базис и континуальную мощность множества всех замкнутых классов [3].

Теорема 7. Среди классов графов, замкнутых относительно операчий бинарной склейки, предполными в классе $\mathscr{G}$ всех графов являются лишь тривиальные классы $\mathscr{G} \backslash\left\{K_{1}\right\}, \mathscr{G} \backslash\left\{C_{1}\right\}$ $u \mathscr{G} \backslash\left\{K_{2}\right\}$.

Доказательство. Покажем сначала, что каждый предполный класс, замкнутый относительно операций бинарной склейки, является тривиальным в надклассе. Предположим, что некоторому предполному классу $P_{1}$ не принадлежат два графа $G_{1}$ и $G_{2}$ из надкласса $P_{2}$. Если $\left|V\left(G_{1}\right)\right|<\left|V\left(G_{2}\right)\right|$ или $\left|E\left(G_{1}\right)\right|<\left|E\left(G_{2}\right)\right|$, то граф $G_{1}$ нельзя реализовать, используя граф $G_{2}$, поскольку графы-операнды бинарной склейки изоморфны подграфам результирующего графа. Если $\left|V\left(G_{1}\right)\right|=\left|V\left(G_{2}\right)\right|$ и $\left|E\left(G_{1}\right)\right|=\left|E\left(G_{2}\right)\right|$, то по той же причине ни один из них нельзя получить, исходя из другого. Таким образом, $\left|P_{2} \backslash P_{1}\right|=1$, то есть $P_{1}$ тривиальный предполный класс.

Граф $G \in P_{2} \backslash P_{1}$ не реализуем с помощью нетривиальной операции бинарной склейки. Следовательно, $|E(G)| \leqslant 1$ и $|V(G)| \leqslant 2$. В $\mathscr{G}$ этим условиям удовлетворяют лишь графы $K_{1}, C_{1}$ и $K_{2}$.

Теорема 7 доказана. 
Таблица 1. Сводная информация о структуре замкнутых классов

\begin{tabular}{|l|c|c|l|}
\hline \hline \multirow{2}{*}{ Операции } & \multicolumn{2}{|c|}{ Число замкнутых классов } & \multirow{2}{*}{ Способы порождения классов } \\
\cline { 2 - 3 } & всех & предполных & \\
\hline \hline склейки и расклейки & 2 & 1 & счетное число одноэлементных базисов \\
\hline склейки и удаления петли & 3 & 1 & счетное число одноэлементных базисов \\
\hline $\begin{array}{l}\text { бинарной склейки, } \\
\text { удаления ребра или петли }\end{array}$ & $\aleph_{0}$ & 2 & $\begin{array}{l}\text { счетное число базисов } \\
\text { с не более чем 3 элементами }\end{array}$ \\
\hline склейки & $2^{\aleph_{0}}$ & 2 & нет базиса или имеется счетное число базисов \\
\hline бинарной склейки & $2^{\aleph_{0}}$ & 3 & единственный конечный или бесконечный базис \\
\hline \hline
\end{tabular}

Из доказательства теоремы 7 вытекает следующее утверждение.

Следствие 8. Каждый предполный класс, замкнутый относительно операчий бинарной склейки, является тривиальным в надклассе.

\section{Список литературы}

1. Яблонский С. В., Введение в дискретную математику. Наука, Москва, 1986.

2. Зыков А. А., Введение в теорию конечных графов. Наука, Москва, 1976.

3. Иорданский М. А., Конструктивные описания графов. Дискретный анализ и исследование операчий (1996) 3, №4, 35-63.

Статья поступила 28.10.2002. 